\title{
Towards Understanding the Mechanisms behind Templated Growth of 2D Magnetite Platelets via Bio-Inspired Approaches
}

$\underline{\text { Bernette M. Oosterlaken }}^{1,2,3}$, Giulia Mirabello ${ }^{1,2,3}$, Joseph P. Patterson ${ }^{4}$ Heiner Friedrich $^{1,2,3}$ and Nico A.J.M. Sommerdijk ${ }^{1,2,3}$

1. Eindhoven University of Technology, Laboratory of Materials and Interface Chemistry, Eindhoven, the Netherlands.

2. ICMS Institute for Complex Molecular Systems, Eindhoven, the Netherlands.

3. CMEM Centre for Multiscale Electron Microscopy, Eindhoven, the Netherlands.

4. Department of Chemistry, University of California, Irvine, Irvine, C 92697, USA.

Magnetite, $\mathrm{Fe}_{3} \mathrm{O}_{4}$, is a naturally occurring iron oxide displaying excellent mechanical and magnetic properties. Its magnetic properties depend on crystal size and shape. In living organisms, the formation of magnetite is precisely regulated, as controlled size and shape crystals are specifically tuned depending on the biological function, even at ambient and aqueous conditions.

Inspiration for this project was found in magnetotactic bacteria, where specialized vesicles with associated transmembrane proteins are directing nucleation and growth of the magnetite crystals. Achieving a similar level of control over crystal shape and size thus far has been challenging in synthetic procedures and the processes behind templated magnetite mineralization are still poorly understood.

Little work has been done on templated magnetite formation so far. In a bio-inspired approach, templated magnetite growth will be investigated, to precisely tune the size and shape of the magnetite crystal. The targeted crystal shape in this project is $2 \mathrm{D}$ platelets. 2D platelets of magnetite may expose unique magnetic state such as vortices, which confine magnetization into a plane. Such magnetic materials might find applications in the field of data storage, among others [1].

A suitable template to direct magnetite growth into $2 \mathrm{D}$ crystals is collagen. Collagen is known to template the formation of calcium phosphate into 2D platelets, like in bone formation, but also calcite $\left(\mathrm{CaCO}_{3}\right)$ and lepidocrocite $(\gamma-\mathrm{FeOOH})$ can be templated into $2 \mathrm{D}$ crystals [2]. To the best of our knowledge, we will be the first to explore the possibilities of growing magnetite in a collagen template.

To template magnetite inside a collagen matrix, magnetite formation outside the collagen template should be inhibited. The addition of polymers to assist in mineral infiltration has been explored for the mineralization of calcium phosphate in collagen. For magnetite, acidic (bio-)molecules, like polypeptides could be used to facilitate infiltration of the precursor phase into the collagen template, followed by intra-mineralization. We will explore the use of polymers and polypeptides as inhibiting agents in the bulk solution to facilitate mineralization within the collagen template. Preliminary collagen mineralization experiments show that an amorphous phase has formed outside the collagen fibrils (Figure 1).

Combining spectroscopic techniques such as Raman spectroscopy with advanced electron microscopy techniques will provide us with new insights in the mechanisms behind magnetite formation inside the template. CryoTEM already has been shown to be of great value when addressing magnetite formation mechanisms [3]. While cryoTEM does not provide any information on the dynamics of the mineralization processes, liquid phase electron microscopy (LP-EM) allows to visualize the processes in-situ and therefore is an appealing complementary technique to CryoTEM. 
The liquid cells for LP-EM provide a confined environment for crystal growth. Therefore, next to the templated magnetite growth inside an organic template, nucleation and growth of magnetite inside the liquid cell will be investigated. The effects of confinement on the crystal shape and size, as well as the effects of the electron beam, are important aspects that will be addressed.

The silicon nitride windows of the liquid cell allow for functionalization with macromolecules. Such macromolecules involve charged polymers or polypeptides, which might induce surface directed magnetite growth. This may lead to better insights in the role of charged polymers during nucleation, nucleation inhibition and crystal growth. Furthermore, the surface functionalization might allow for directing the shape and size of the magnetite crystals.

Preliminary experiments on growing magnetite in the confined space of a liquid cell suggests that magnetite indeed has formed inside the liquid cell. The experimental conditions need to be optimized, after which the formation of magnetite could be visualized with LP-EM.

\section{References:}

[1] A Fert et al, Nature Nanotechnology 8 (2013), p. 152.

[2] Y Xu et al, in preparation (2018).

[3] J Baumgartner et al, Nature Materials 12 (2013), p. 310.
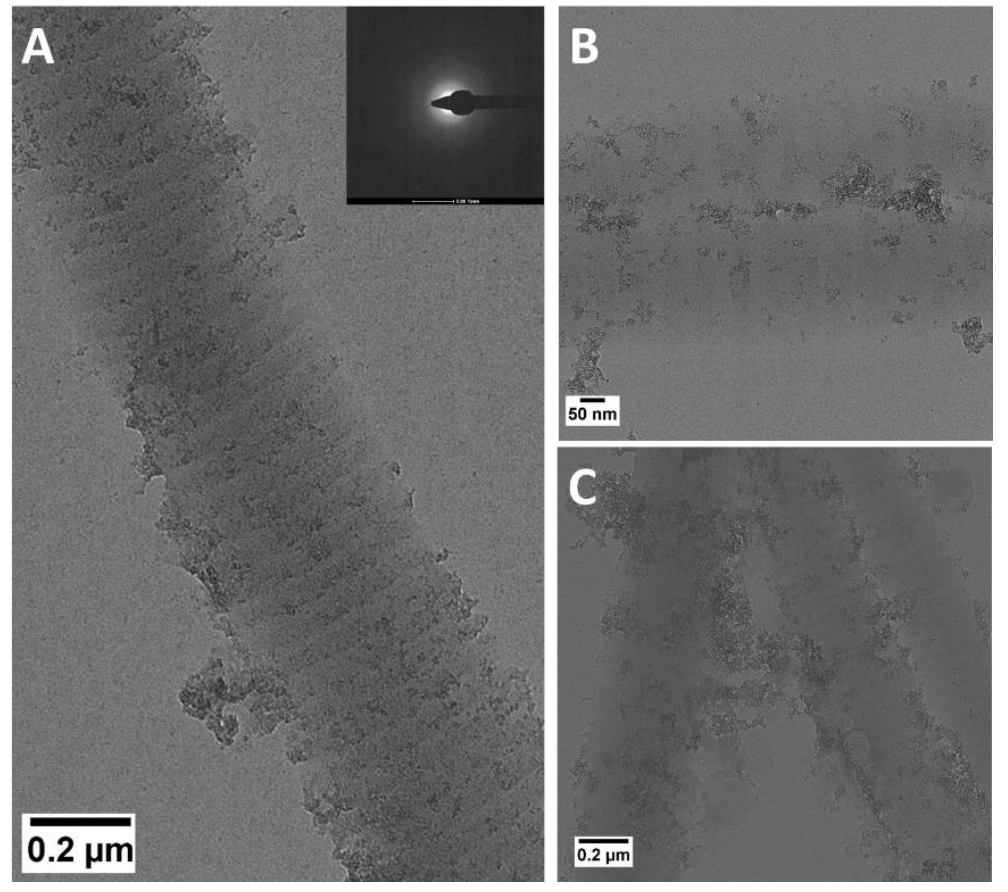

Figure 1. Dry TEM on collagen fibrils (Bovine Achilles, type I) mineralized in the presence of poly(aspartic acid) (ratio Fe: Asp $1: 5$ ). An amorphous phase has formed around the collagen fibrils. A : Scalebar : $0.2 \mu \mathrm{m}$. Inset : Selected area electron diffraction of A. B : Scalebar : $50 \mathrm{~nm}$. C : Scalebar : $0.2 \mu \mathrm{m}$. 